\title{
Small scale tests of cement with focused ion beam and nanoindentation
}

\author{
Jiři Němeček ${ }^{1, *}$, Jan Maňák ${ }^{2}$, Tomáš Krejčí ${ }^{1}$ and Jiří Němeček ${ }^{1}$ \\ ${ }^{1}$ Czech Technical University in Prague, Faculty of Civil Engineering, Prague, Czech Republic \\ ${ }^{2}$ Academy of Sciences, Institute of Physics, Prague, Czech Republic
}

\begin{abstract}
Nanoindentation is used for characterization of small scale material properties of hydrated cement. It is employed as a precise loading tool on samples fabricated with Focused Ion Beam milling (FIB). The effect of heat on the microstructure of cement during different FIB energy loads is studied. Milling currents as low as $0.1 \mathrm{nA}$ can be considered as save and not damaging. Micrometer sized beams were bent to reveal strength and fracture characteristics. Small scale elastic properties, tensile strength and fracture energy of individual low scale microstructural constituents of cement paste like C-S-H rich phases and Portlandite were assessed. Very high tensile strengths at the micrometer scale were observed for cement paste hydration products (200-700 MPa) with fracture energies $4-20 \mathrm{~J} / \mathrm{m}^{2}$ The results are consistent with atomistic simulations and multi-scale modeling from available literature.
\end{abstract}

\section{Introduction}

Tensile strength and fracture toughness are very important characteristics of cement based materials. Cementitious composites are based on cement matrix which is characterized by a brittle failure and the ductility of the whole composite specimen depends very much on the its size, volume content and shape of defects that are present in the form of cracks and voids. From the macroscopic point of view the size dependence and strength scaling has been studied by fracture mechanics for a long time. Energetic size effect on strength was derived by Bažant [1]. However, the mechanisms of the fracture and related micromechanical characteristics in the fracture process zone remained. It is now clear that the initiation of the fracture happens on scales much smaller than the lab specimens, i.e. on the microscale. Thus, advanced material models (usually multi-scale) must take into account intrinsic strength and fracture properties of individual small scale constituents of the cementitious matrix.

With the development of novel experimental techniques like electron microscopy, nanoindentation [2] and focused ion beam milling (FIB) [3] it is now possible to extract properties on scales as low as $1 \mu \mathrm{m}$. While microscopy enables visualizing morphology and chemical composition of microstructural features of cement matrix nanoindentation allows micromechanical characterization of the small material volumes. Elastic and creep

\footnotetext{
*Corresponding author: jiri.nemecek@ fsv.cvut.cz
} 
properties were already found [4, 5]. But until recently, it was not possible to access fracture properties of the small scale constituents. Němeček et al. developed a methodology based on bending of FIB prepared micrometer sized specimens [6] where FIB allows preparation of geometrically precise microscopic samples and nanoindenter allows precise measurement of the bending response of the micro-beams.

The work presented in this paper presents FIB preparation technique, quantifies possible FIB caused damage to the samples and shows the strength and fracture properties of individual constituents of cement paste at the micrometer scale.

\section{Methodology and experiments}

First, samples were scanned in SEM and locations of individual microstructural phases detected. Then, micro-beams were milled with FIB (FEI Quanta 3D FEG) in the form cantilevers (15-20 $\mu \mathrm{m}$ long with triangular cross-section 3-4 $\mu \mathrm{m})$, Fig. 1. As the next step, micro-beams were loaded at the free end by nanoindenter (Hysitron TI-700) and bent. Bending response of the beams was tested in several elastic cycles. Standard beam theory was used to calculate Young's modulus as

$$
E=\frac{F L^{3}}{3 w I_{y}},
$$

where $F$ and $w$ are the force and deflection reached in the elastic regime, respectively, $L$ is the micro-beam length, $I_{y}$ the second moment of inertia of the cross section. Then beams were bent until the break and tensile strength and fracture energy calculated according to the methodology used in 6 . From load-deflection curves measured by nanoindenter tensile strength, $f_{t}$, and supremum fracture energy, $G_{f}$, were calculated as

$$
\begin{array}{r}
f_{t}=\frac{F_{\max } L}{W_{h}}, \\
G_{f}^{\text {sup }}=\frac{1}{A_{f}} \int_{0}^{w_{\max }} F d w,
\end{array}
$$

where $F_{\max }$ is the maximum measured force, $W_{h}$ is the section modulus, $w_{\max }$ is the peak deflection and $A_{f}$ is the nominal fracture area (i.e. $A_{f}=1 / 2 b d$ for the triangle $b \times d$ ). Measurement of the load-displacement curve was done in the displacement controlled regime. However, current capabilities of the instrument do not allow to capture softening and the descending branch of the curves is not stable. Thus, the fracture energy was, in accordance with [6] assessed as supremum energy assuming that micro-beam behavior shows neither snap-back nor softening.

\subsection{Samples}

Samples of cement paste made from CEM-I 42.5R mixed with water to binder ratio 0.4 were produced and stored in water for 8 years to support full hydration. Microstructure of the samples was scanned by electron microscope (SEM) and approximate volumetric content of individual microstructural phases calculated by image analysis. The phases included inner product $(\sim 28 \%)$, outer product $(\sim 50 \%)$, Portlandite crystals $(\mathrm{CH})(\sim 10 \%)$, unhydrated clinkers $(3 \%)$ and capillary porosity $(12 \%)$, see [6] for details. Prior to further 
analyses samples were cut into $5 \mathrm{~mm}$ thick slices and prepared with a metallographic procedure [4-6] to get a flat and smooth surface with small roughness of several tens of nm.

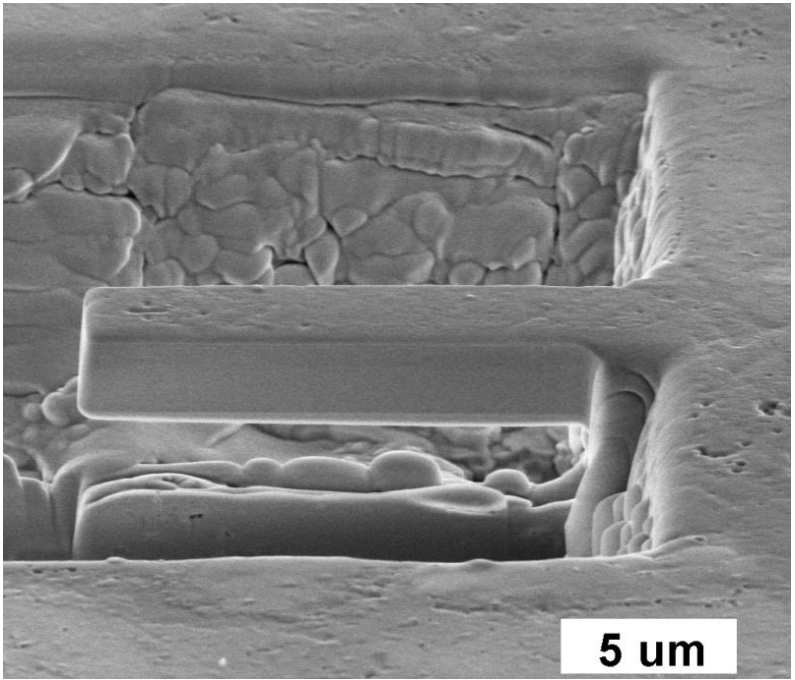

Fig. 1. FIB milled micro-beam.

\subsection{Focused Ion Beam milling}

Micro-beams' geometry with triangular cross section was already optimized in [6] to be feasible for fabrication, small enough to represent a particular phase and large enough to minimize geometrical imperfections. The FIB interaction with the sample causes generation of the heat that can further damage the sample. To study such effect, three energy levels were tested: high energy (HE), standard energy (SE) and low energy (LE). Common acceleration voltage of $30 \mathrm{kV}$ was used for all samples. The milling current varied and was 30, 1 and $0.1 \mathrm{nA}$ for HE, SE and LE variants, respectively. Details on the FIB milling steps can be found [12]. It can be seen in Fig. 2 that LE milling produces fine undamaged samples while HE milling causes rounded geometry with some artifacts and damage on the sample surface.
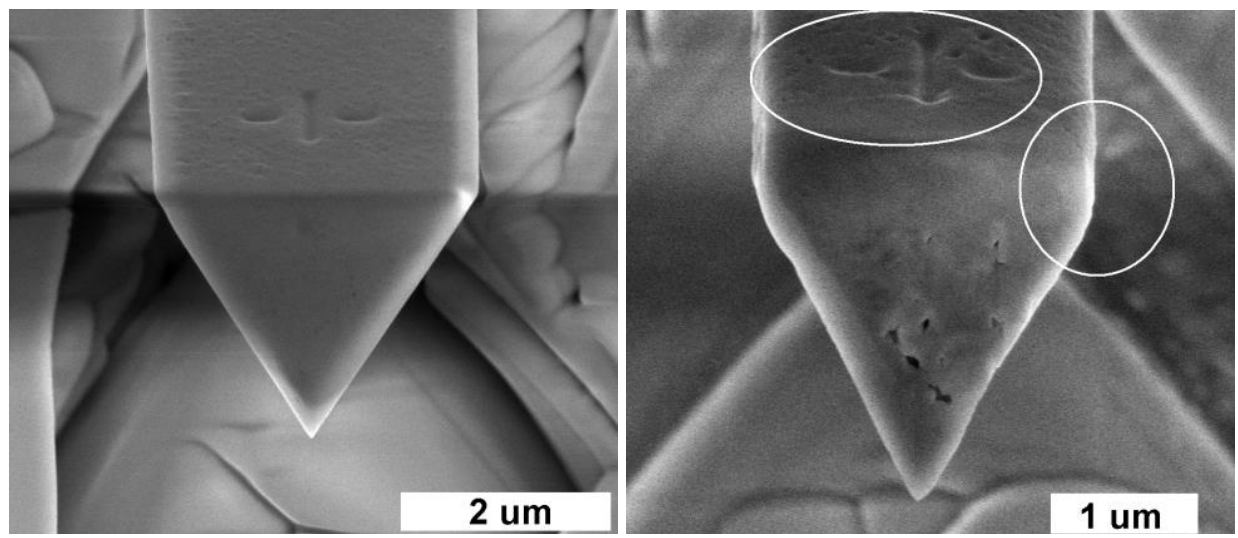

Fig. 2. Cross section of micro-beams milled with different energies LE (left), HE (right). 


\subsection{Heat transfer model of the FIB process}

In order to quantify the effect of temperature rise in the micro-beams a finite element (FE) model was proposed. The non-stationary heat transfer on a domain much larger with respect to the beam dimensions was solved. An axisymmetrical FE mesh of $5 \times 5 \mathrm{~mm}$ fillled with quadrilateral elements with linear approximations was created, Fig. 3. The smallest element size at the point of ion beam contact was $100 \mathrm{~nm}$.

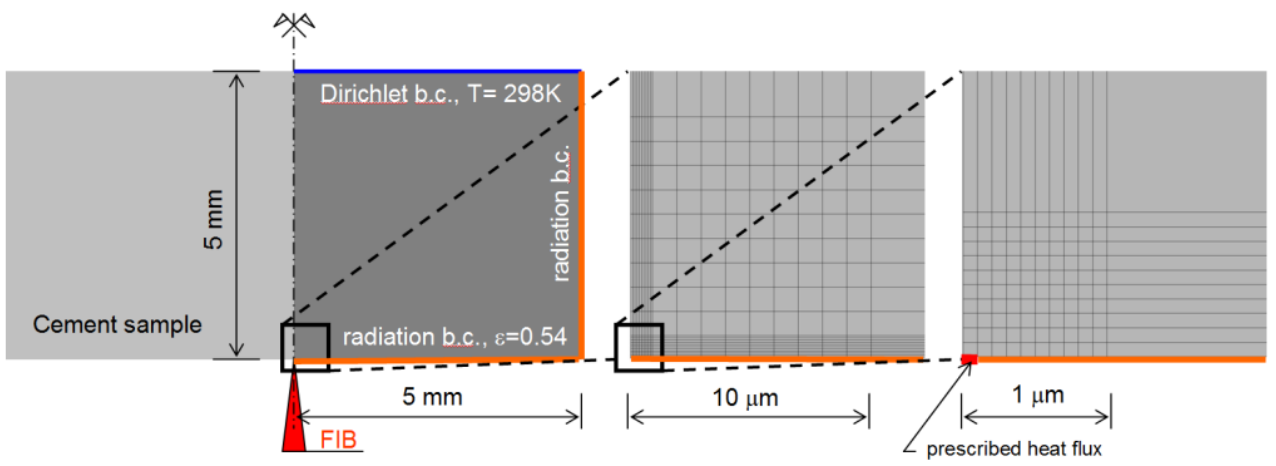

Fig. 3. Scheme of the heat transfer FE model.

\section{Results and discussion}

Temperature calculations (Fig. 4) showed that SE milling causes temperature rise around $100{ }^{\circ} \mathrm{C}$ at the point of FIB contact and about $40{ }^{\circ} \mathrm{C}$ in $100 \mathrm{~nm}$ distance. On the other hand, HE milling causes extreme temperatures above $3000{ }^{\circ} \mathrm{C}$ at the point of contact leading to inevitable micro-structural changes. The zone with temperatures above $100{ }^{\circ} \mathrm{C}$ is still about $2 \mu \mathrm{m}$ away from the contact point. It must be noted that the temperature is very high but not fully real since the model does not take into account other effects like heat sinks during evaporation and phase changes. LE milling does not show temperatures above $100{ }^{\circ} \mathrm{C}$ in any point of the domain and can be considered as save and not damaging to the material. Such results agree with microstructural observation in SEM.
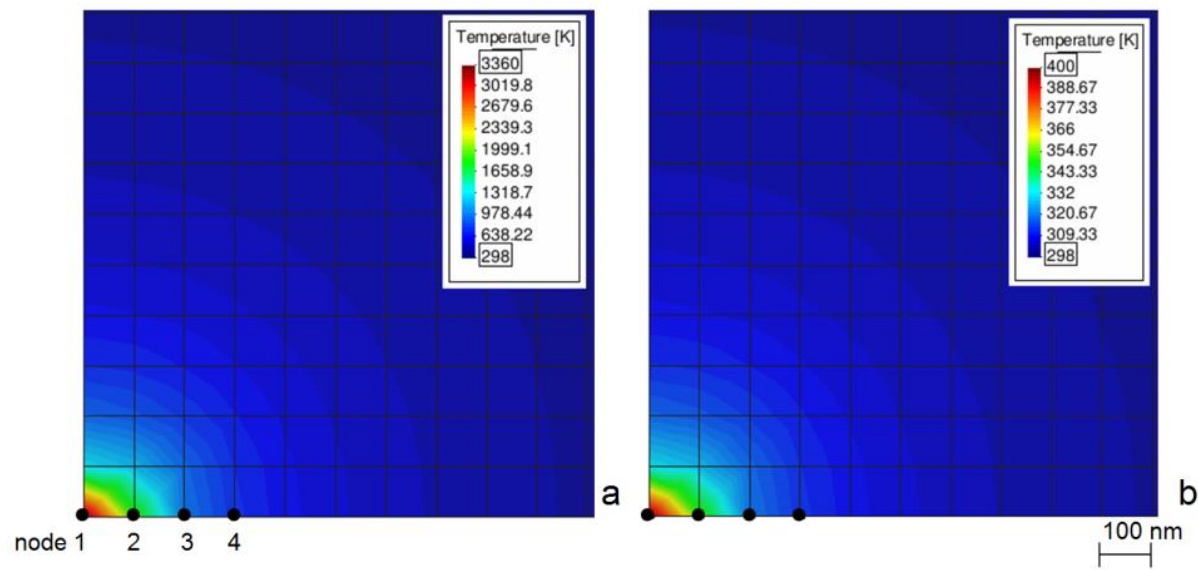

Fig. 4. Calculated temperatures around the point of beam contact HE (left), SE(right).

Load-deflection curves were obtained on 7-10 micro-beams for each studied phase. Mechanical parameters of the phases are summarized in Tab. 1. Fig. 5 depicts loading 
diagrams normalized with respect to beam length. Behavior of all phases was mostly linear upto the break with sudden descending branch which is a manifestation of their brittle nature and unability of the instrumnet to capture softening, as mentioned before. The results of elastic properties are in good agreement with literature data of specific microscale constituents. Tensile strengths are much higher than macroscale values of cement paste showing a high scaling factor. The discrepancy between the scales is explained by multiscale models that take into account defect present at higher composite levels [7]. The defects are voids, crakcs or inclusions. Strength calculation at $\mathrm{C}-\mathrm{S}-\mathrm{H}$ level provided by molecular dynamics models yields tensile strength for low density and high density C-S-H as 550-720 MPa [8]. AFM measurements revealed $930 \mathrm{MPa}$ [9]. The values are comparable with microbending tests in Tab. 1. MD simulations give fracture energies in the range of $0.4-3 \mathrm{~J} / \mathrm{m}^{2}[10,11]$ which is a bit less than our supremum energies.

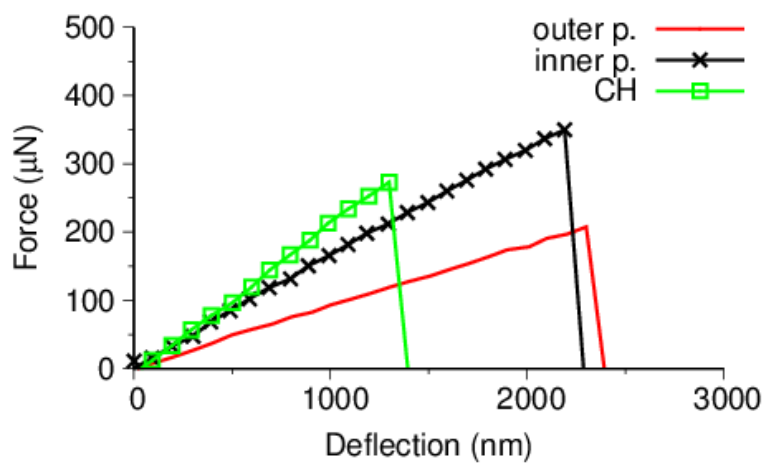

Fig. 5. Examples of load-deflection diagrams of micro-beams in different phases.

Table 1. Micromechanical properties derived from micro-beam bending.

\begin{tabular}{|l|l|l|l|}
\hline & \multicolumn{1}{|c|}{$\begin{array}{c}\text { Outer } \\
\text { product }\end{array}$} & \multicolumn{1}{|c|}{ Inner product } & CH \\
\hline$E(\mathrm{GPa})$ & $24.9 \pm 1.3$ & $33.6 \pm 2.0$ & $39.0 \pm 7.1$ \\
\hline$f_{t}(\mathrm{MPa})$ & $\begin{array}{l}264.1 \pm \\
73.4\end{array}$ & $700.2 \pm 198.5$ & $655.1 \pm 258.3$ \\
\hline$G_{f}^{\text {sup }}\left(\mathrm{J} / \mathrm{m}^{2}\right)$ & $4.4 \pm 1.9$ & $19.7 \pm 3.8$ & $19.9 \pm 14.4$ \\
\hline
\end{tabular}

\section{Conclusions}

The FE model of the FIB milling process indicates that high temperatures develop in cement paste for high energy dose. For $30 \mathrm{kV}$ and $30 \mathrm{nA}$ the extent of temperature affected zone (over $100{ }^{\circ} \mathrm{C}$ ) is about $2 \mu \mathrm{m}$ which is in the same scale as the micro-beam dimensions. The standard energy level $(30 \mathrm{kV}, 1 \mathrm{nA})$ can affect about $10 \%$ of the beam cross section and the lowest energy level $(30 \mathrm{kV}, 0.1 \mathrm{nA})$ can be considered as safe causing temperatures below $100{ }^{\circ} \mathrm{C}$ and negligible damage.

It was found that the tensile strength of microstructural constituents of cement paste is two orders of magnitude higher than the macroscopic value (260-700 MPa, Tab. 1). Such results are in relation with molecular dynamics simulations available from literature 
$[8,10,11]$. The strength scaling corresponds to the multi-scale character of the composite in which defects (in the form of cracks or voids) occur at higher levels. Multi-scale models can separate the scales and explain such situation [7].

Financial support of the Czech Science Foundation (project 17-05360S) and the Czech Technical University in Prague (SGS18/114/OHK1/2T/11) is gratefully acknowledged.

\section{References}

1. Bažant, Z. P., Scaling of Structural Strength, second ed., (Elsevier Ltd., 2005).

2. Fischer-Cripps A.C., Nanoindentation, (Springer, 2002).

3. Gianuzzi, L. and Stevie, F., Introduction to Focused Ion Beams. Instrumentation, Theory, Techniques and Practice, (Springer, 2005).

4. Němeček, J., Králík, V. and Vondřejc, J., Cem Concr Comp 36 (2013), 85-92.

5. Němeček, J., Šmilauer V., Kopecký, L., Cem Concr Comp 33 (2011), 163-170

6. Němeček, J., Králík, V., Šmilauer V., Polívka L., Jager, A., Cem Concr Comp 73 (2016), 164-173.

7. Němeček, J., Šmilauer V., Němeček, J., Kolařík, F., Proceedings of EURO-C 2018, (Taylor and Francis, 2018).

8. Davie, C. and Masoero, E., 10th International Conference on Mechanics and Physics of Creep, Shrinkage, and Durability of Concrete and Concrete Structures, 2015.

9. Plassard, C., Lesniewska, E., Pochard, I. and Nonat A., Langmuir 21(16) (2005), 7263-7270.

10. Ghebrab, T., Soroushian, P., J. Concr. Struct. Mater. 7 (4) (2010), 37-43.

11. Bauchy, M., Laubie, H., Qomi, M.A., Hoover, C., Ulm, F.-J., Pelenq, R.-M., J., Non Cryst. Solids 419 (2015), 58-64.

12. Němeček, J., Maňák, J., Němeček, J., Krejčí, T., Cem Concr Comp 100 (2019), 139149. 